Elsevier

PAI 01242

\title{
Sleep fragmentation in the arthritic rat
}

\author{
Carol A. Landis *, Connie R. Robinson *** and Jon D. Levine ** \\ Departments of * Physiological Nursing. Medicine and ** Oral and Maxillofacial Surgery, Schools of Nursing, Medicine and Dentistry, \\ University of California, San Francisco, San Francisco, CA 94143-0604 (U.S.A.), and *** School of Nursing, University of Michigan, \\ Ann Arbor, MI 48109-0482 (U.S.A.)
}

(Received 4 January 1988, accepted 16 February 1988)

\begin{abstract}
Summary We examined the diurnal sleep-wake patterns in the adjuvant arthritic rat. In contrast to control rats, arthritic rats lacked a normal diurnal variation in sleep and wakefulness. Thus, arthritic rats exhibited no differences in the mean number or duration of bouts of sleep and episodes of wakefulness between light and dark hours. Arthritic rats also had a marked increase in the fragmentation of their sleep manifested by an increased number of sleep bouts and episodes of wakefulness and a decrease in the duration of episodes of deep sleep recorded both during the time of maximal sleep (08.00-11.00 h) and of maximal wakefulness $(20.00-23.00 \mathrm{~h})$. The possibility that the experience of chronic pain causes these marked changes in sleep patterns in the arthritic rat is discussed.
\end{abstract}

Key words: Chronic pain; Experimental pain; Adjuvant arthritis; Sleep

\section{Introduction}

Patients with arthritis frequently complain of difficulty falling asleep and of frequent awakenings during the night $[21,34]$. Electroencephalogram (EEG) studies of sleep patterns show that patients with rheumatoid arthritis take a longer time to fall asleep, have reduced sleep, and have frequent episodes of awakening after sleep onset $[27,30]$. They have an additional sleep disturbance characterized on the EEG by the presence of alpha frequency waveforms during non-rapid eye movement (NREM) sleep stages [27,30]. This nocturnal EEG arousal pattern, called alphaNREM sleep, has been associated with reports of increased pain, tenderness, weakness, and fatigue

Correspondence to: Ion D. Levine, M.D., Ph.D., Division of Rheumatology, U-426, Box 0724, University of California, San Francisco, San Francisco, CA 94143, U.S.A.
[30]. It is unclear whether these sleep disturbances associated with theumatoid arthritis are caused by pain, and/or by other aspects of this systemic disease. For example, periodic extremity movements [27] and psychological distress also cause disrupted sleep [21] and may contribute to the abnormal sleep patterns in patients with arthritis. Thus, it has been difficult to establish in patients with arthritis that pain is a cause of their sleep disturbances.

Adjuvant arthritis in the rat, a disease model of chronic pain $[7,8]$, is associated with hyperalgesia $[7,8,10,17,18,23,26,33]$. The hyperalgesia is highest when pain-related behaviors are most pronounced $[7,17]$. Arthritic rats have significantly more sleep preparatory (resting) behaviors without a significant increase in sleep [17]. These data suggest that arthritic rats may have a fragmented pattern of sleep similar to that observed in patients with rheumatoid arthritis. The possibility that pain may cause disrupted sleep in arthritic rats is suggested 
by the fact that the elimination of pain in these rats reduces signs of morbidity [14]. Although many studies document dramatic changes in the behavior of arthritic rats, no studies have quantified changes in EEG sleep patterns. In the present study we describe a loss of diurnal variations in sleep and wake stages and a fragmentation in sleep patterns in rats produced by the induction of experimental arthritis.

\section{Methods}

\section{Surgical preparation and recordings}

The experiments were performed on male Sprague-Dawley rats (250-350 g; Bantin and Kingman, Fremont, CA). To record EEG and EEG-theta sleep patterns [2], rats were anesthetized with sodium pentobarbital $(50 \mathrm{mg} / \mathrm{kg})$ and stainless steel wire electrodes were placed on the dura through holes drilled in the skull. To record electromyographic (EMG) activity, 2 silver wires were placed under each temporalis muscle [35]. All electrodes were secured to the skull with dental acrylic. Prior to recording sessions, a cable was attached to the electrodes and the rats were placed in Plexiglas cages $(12 \mathrm{~cm} \times 21 \mathrm{~cm}$ floor, $15 \mathrm{~cm}$ high), located in a sound attenuated recording chamber. The ambient temperature of the Plexiglas cages was $23^{\circ} \mathrm{C}\left( \pm 2^{\circ} \mathrm{C}\right)$. Throughout the experiments, including time in home cages and recording chambers, rats were maintained on a 12 $\mathrm{h}$ light-dark schedule with lights on at $08.00 \mathrm{~h}$. Food and water were available ad lib.

When rats are adapted to this $12 \mathrm{~h}$ light-dark cycle, they typically demonstrate a diurnal pattern of sleep and wakefulness. Rats sleep most during the first $3 \mathrm{~h}$ of the light period $(08.00-11.00)$ and least during the first $3 \mathrm{~h}$ of the dark period (20.00-23.00) [6,29]. Accordingly, sleep and wake patterns were recorded for $3 \mathrm{~h}$ at the beginning of the light period and again at the beginning of the dark period. Rats were allowed 1 week to recover from surgery and 1 day to adapt to the recording cage. After 2 baseline recording days, rats were arthritized. Arthritic rats were allowed to readapt to the recording chamber on the third day after the onset of clinical signs of arthritis and sleep patterns were recorded again for 2 days.

\section{Temperature}

Since changes in body temperature can affect sleep $[20,22]$ and since elevations of body temperature have been reported in arthritic rats [9], core temperature was recorded in 12 non-arthritic and 11 arthritic rats. Rats had a small thermally sensitive transmitter (Mimi-mitter, Model VM, Sun River, OR, U.S.A.) surgically inserted into the peritoneal cavity at the same time that EEG and EMG electrodes were implanted [16]. Body temperature was recorded at $07.00 \mathrm{~h}, 10.00 \mathrm{~h}, 13.00 \mathrm{~h}$, $19.00 \mathrm{~h}$ and $22.00 \mathrm{~h}$ on the days that sleep recordings were made. Core body temperatures recorded at $10.00 \mathrm{~h}$ and $22.00 \mathrm{~h}$, the time of a large difference in body temperature of normal rats [15], were used for statistical comparison.

\section{Scoring of sleep stages}

Polygraph recordings of EEGs were divided into consecutive $30 \mathrm{sec}$ segments of time, called epochs. Each epoch was then scored, blind, into wakefulness or 1 of 4 sleep stages according to the classification of Mistlberger et al. [29]. The latter scales distinguish wakefulness (Wake), low-amplitude NREM sleep (NREM-LS), high-amplitude NREM sleep 1 (NREM-HS1), high-amplitude NREM sleep 2 (NREM-HS2), and paradoxical sleep (PS, also called rapid eye movement (REM) sleep). The waking state was defined by a low-amplitude, fast frequency EEG accompanied by high-amplitude tonic and phasic EMG activity. NREM-LS (a transition stage from wakefulness to sleep and from one sleep stage to another) was differentiated from wakefulness by a decrease in the tonic and an absence of phasic EMG activity. NREM-HS1 was scored as present when the amplitude of the EEG was high and the amplitude of the EMG was low for $30-50 \%$ of a $30 \mathrm{sec}$ segment of time (epoch). NREM-HS2 (also called slowwave sleep) was scored when these same EEG and EMG patterns were present for more than $50 \%$ of an epoch. PS was defined by a low-amplitude EEG, a higher amplitude of EEG-theta activity than that of wakefulness, and the absence of EMG activity, with the exception of occasional muscle twitches.

Sleep bouts and sleep stage episodes were measured for each $3 \mathrm{~h}$ of EEG recordings in order to 
characterize fragmentation in the pattern of sleep. Sleep bouts were uninterrupted periods of sleep time. The number and duration of sleep bouts were measured as consecutive $30 \mathrm{sec}$ epochs containing NREM-LS, NREM-HS1, NREM-HS2, and PS that occurred between at least 2 consecutive epochs (1 $\mathrm{min})$ of wakefulness [29]. Sleep and wake stage episodes are the number of times an individual sleep or wake stage occurred during a 3 $h$ recording period. Stage episodes were measured as the number of times consecutive epochs of each stage were scored during a $3 \mathrm{~h}$ period of EEG recordings. The mean duration of an episode was calculated by dividing the sum of all the epochs of a stage scored in a $3 \mathrm{~h}$ period of time by the number of times that stage occurred in the same time period (the number of episodes of the stage).

\section{Arthritis}

Adjuvant arthritis was induced in all rats by the intradermal injection of $0.15 \mathrm{ml}$ of a $10 \mathrm{mg} / \mathrm{ml}$ suspension of heat-killed Mycobacterium butyricum (Difco, Detroit, MI, U.S.A.) in mineral oil, in the tail [32]. This preparation elicits a bilateral, distal arthritis that usually affects the hind paws more severely than the forepaws. Onset of clinically apparent arthritis (swelling and tenderness in the paws) occurs approximately 2 weeks after the injection of adjuvant. The disease remains active for at least 2 weeks $[7,33]$. The mean onset of arthritis in 7 rats was $13.7 \pm 2.1$ days ( \pm \pm 1 S.D.).

To monitor severity of the disease, a $0-3$ rating scale was used in which $0=$ no signs of swelling, $1=$ minimal, $2=$ moderate, and $3=$ severe swelling [14]. The scores for swelling were measured daily in each paw beginning on post-injection day 10. The score for each paw was summed to yield a daily clinical score of illness severity (range 0-12) for each rat. The daily clinical scores for each rat were averaged for the 2 days that the sleep EEG was recorded. The average clinical severity score in 7 rats was $3.21 \pm 1.8(\mathrm{X} \pm 1$ S.D., range 1.5-7.0). The clinical scores measured on the day following the completion of sleep recordings in arthritic rats correlated significantly $(r=0.8907, P<0.01)$ with scores of severity of joint injury derived from whole body radiographs taken on the same day. Radiographic scores of each paw were determined independently by a skeletal radiologist using the rating scale of Ackerman et al. [1] in which 0 indicated no joint injury, $1=$ mild, $2=$ moderate, and 3 = severe joint injury.

This study followed the IASP guidelines for investigating experimental pain in animals [13]. The duration of the experiment was kept to a minimum and the number of animals used was small. The animals were housed with no more than 2 rats per cage to reduce the possibility of painful contact between them. The floors of the recording cages and home cages were covered with soft saw dust. Food was available at the level of the cage floor, and drinking water was within easy reach.

\section{Statistical analysis}

The paired Wilcoxon signed rank test was used to compare the number and duration of sleep bouts and sleep and wake stage episodes and the paired Student's $t$ test was used to compare core body temperatures in groups of non-arthritic and arthritic rats. Data for sleep variables and temperature are reported as the mean \pm 1 standard deviation (X \pm 1 S.D.).

\section{Results}

\section{Diurnal variations in sleep patterns}

Non-arthritic rat. Non-arthritic rats had longer periods of uninterrupted time asleep during light than dark hours. Although the total number of sleep bouts did not differ significantly between the light $(8.6 \pm 2.95)$ and dark $(7.43 \pm 2.3)$ hours in non-arthritic rats (Fig. 1A), the mean duration in minutes of a sleep bout was significantly longer in the light $(13.98 \pm 3.76 \mathrm{~min})$ than the dark $(8.56 \pm$ $2.72 \mathrm{~min}, P<0.03$ ) hours (Fig. 1B). Non-arthritic rats also had significantly more episodes of highamplitude NREM sleep stages (NREM-HS1 and NREM-HS2) and of paradoxical sleep (PS) in light hours (all $P<0.05$, Table I). In addition, the duration of NREM-HS2 sleep stage episodes was twice as long in the light hours $(P<0.05)$. The number of wake episodes was not significantly different between light and dark hours but the 

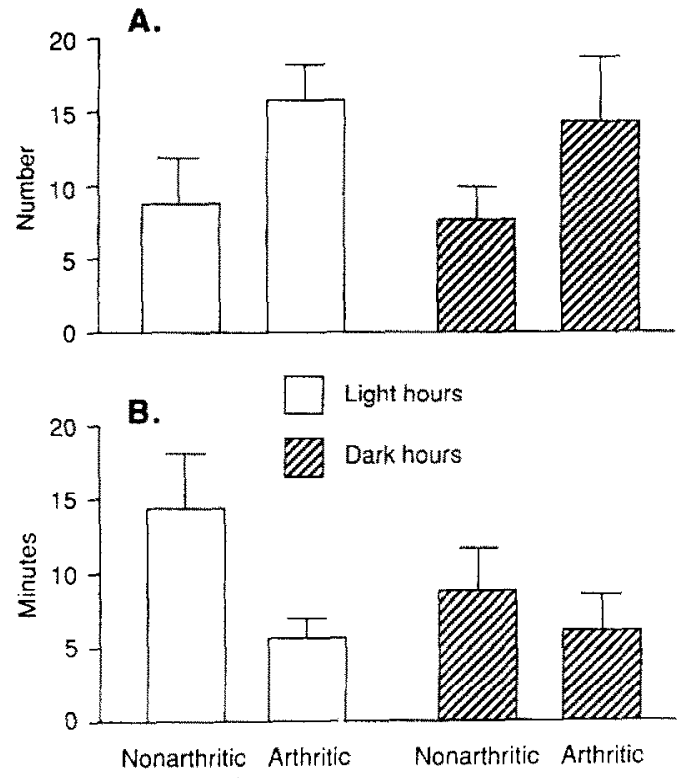

Fig. 1. A: the number of sleep bouts during $3 \mathrm{~h}$ polygraph recordings in light $(08.00-11.00 \mathrm{~h})$ and dark $(20.00-23.00 \mathrm{~h})$ hours ( $\mathrm{n}=7$ rats). B: the mean duration of sleep bouts in minutes for the same time periods. Sleep bout duration = sum of total time in all sleep bouts/total number of sleep bouts in a $3 \mathrm{~h}$ period.

mean duration of wake episodes was significantly longer in the dark hours ( $P<0.02$, Table II).

Arthritic rat. In comparison to the non-arthritic rats, the arthritic rats did not have significant differences in the number and/or mean duration of sleep bouts between the light $(15.5 \pm 2.3 ; 5.48$ $\pm 1.28 \mathrm{~min})$ and dark hours $(13.98 \pm 4.4 ; 5.9 \pm$ $2.41 \mathrm{~min}$, Fig. 1). Furthermore, arthritic rats did not have significant differences between light and dark hours in the number (Table I) and duration (Table II) of episodes of sleep stages and of wakefulness.

\section{Comparison of arthritic and non-arthritic rat}

The sleep patterns of arthritic rats were more fragmented than those of non-arthritic rats. Thus, arthritic rats had twice as many sleep bouts as non-arthritic rats during both the light (arthritic $15.5 \pm 2.3$, non-arthritic $8.6 \pm 2.95, P<0.02$ ) and the dark (arthritic $13.98 \pm 4.4$, non-arthritic 7.43 $\pm 2.3, P<0.03$ ) hours (Fig. 1A). The mean duration of these bouts of sleep in arthritic rats was also significantly shorter in light hours (arthritic $5.48 \pm 1.3 \mathrm{~min}$, non-arthritic $13.98 \pm 3.8 \mathrm{~min}, P<$ 0.02 , Fig. 1B). Arthritic rats also had a significant increase in the number of wakefulness episodes during both the light and dark $(P<0.05$ and $P<0.03$, respectively) hours (Table $\mathrm{I}$ ) and a significant reduction in the duration of these episodes in the dark hours ( $P<0.03$, Table II). This fragmentation of sleep in arthritic rats was further manifested by a significant reduction in the duration of episodes of high-amplitude NREM sleep 2 (slow-wave sleep) during light hours $(P<0.02$, Table II), and a significant increase in the number

\section{TABLE I}

\section{STAGE EPISODE (number)}

$\mathrm{n}=7$ rats, mean number of stage episodes \pm S.D. Statistical comparisons are indicated by pairs of letters. Paired Wilcoxon signed rank test, all $P<0.05$.

\begin{tabular}{|c|c|c|c|c|c|}
\hline & Wake & NREM-LS & NREM-HS1 & NREM-HS2 & PS \\
\hline \multicolumn{6}{|l|}{ Light period } \\
\hline Non-arthritic & $\begin{array}{l}16.6 \\
\pm 4.4(\mathrm{a})\end{array}$ & $\begin{array}{l}13.96 \\
\pm 4.5(\mathrm{c})\end{array}$ & $\begin{array}{l}35.8 \\
\pm 9.0(\mathrm{e})\end{array}$ & $\begin{array}{l}32.9 \\
\pm 6.7(\mathrm{~g})\end{array}$ & $\begin{array}{l}11.8 \\
\pm 4.4(h, i)\end{array}$ \\
\hline Arthritic & $\begin{array}{l}25.6 \\
\pm 4.2(\mathrm{a})\end{array}$ & $\begin{array}{l}21.9 \\
\pm 3.1 \text { (c) }\end{array}$ & $\begin{array}{l}35.2 \\
\pm 5.2\end{array}$ & $\begin{array}{l}26.7 \\
\pm 5.8\end{array}$ & $\begin{array}{l}5.5 \\
\pm 2.2(\mathrm{i})\end{array}$ \\
\hline \multicolumn{6}{|l|}{ Dark period } \\
\hline Non-arthritic & $\begin{array}{l}13.4 \\
\pm 3.7(b)\end{array}$ & $\begin{array}{l}11.1 \\
\pm 4.5(\mathrm{~d})\end{array}$ & $\begin{array}{l}23.9 \\
+5.98(\mathrm{e}, \mathrm{f})\end{array}$ & $\begin{array}{l}19.86 \\
\pm 4.47(\mathrm{~g})\end{array}$ & $\begin{array}{l}6.86 \\
\pm 3.34(h)\end{array}$ \\
\hline Arthritic & $\begin{array}{l}24.26 \\
\pm 4.2(\mathrm{~b})\end{array}$ & $\begin{array}{l}19.3 \\
\pm 3.6(\mathrm{~d})\end{array}$ & $\begin{array}{l}35.9 \\
+5.9(f)\end{array}$ & $\begin{array}{l}23.5 \\
\pm 7.7\end{array}$ & $\begin{array}{l}8.27 \\
\pm 3.7\end{array}$ \\
\hline
\end{tabular}


TABLE II

STAGE EPISODE DURATION (min)

$\mathrm{n}=7$ rats, mean episode duration \pm S.D. Statistical comparisons are indicated by pairs of letters. Paired Wilcoxon signed rank test, all $P<0.05$.

\begin{tabular}{llllll}
\hline & Wake & NREM-LS & NREM-HS1 & NREM-HS2 & PS \\
\hline Light period & & & & & \\
Non-arthritic & 4.3 & 0.62 & 0.74 & 2.3 & 1.89 \\
& $\pm 0.81(\mathrm{a})$ & $\pm 0.12(\mathrm{c})$ & $\pm 0.07(\mathrm{~d})$ & $\pm 0.53(\mathrm{e}, \mathrm{f})$ & \pm 0.29 \\
Arthritic & 4.57 & 0.78 & 0.85 & 1.08 & 1.85 \\
& \pm 1.2 & $\pm 0.11(\mathrm{c})$ & \pm 0.22 & $\pm 0.19(\mathrm{f})$ & \pm 0.38 \\
Dark period & & & & \\
Non-arthritic & 9.06 & 0.67 & 0.90 & 1.06 & 1.64 \\
& $\pm 2.18(\mathrm{a}, \mathrm{b})$ & \pm 0.10 & $\pm 0.13(\mathrm{~d})$ & $\pm 0.22(\mathrm{e})$ & \pm 0.19 \\
Arthritic & 4.83 & 0.69 & 0.75 & 0.92 & 1.65 \\
& $\pm 1.35(\mathrm{~b})$ & \pm 0.08 & \pm 0.08 & \pm 0.16 & \pm 0.24 \\
\hline
\end{tabular}

of episodes of high-amplitude NREM sleep 1 during the dark hours $(P<0.05$, Table I). In addition, arthritic rats had a significant increase in low-amplitude NREM sleep stage episodes during both the light and dark hours (both $P<0.02$, Table I). However, there were no significant differences in the duration of these episodes $(P>$ $0.05)$.

\section{Temperature variations}

Arthritic rats had a small, albeit significant, elevation in core temperature recorded at $10.00 \mathrm{~h}$ (arthritic rats, $39.6^{\circ} \mathrm{C} \pm 0.592, \mathrm{n}=11$, and nonarthritic rats, $38.9^{\circ} \mathrm{C} \pm 0.61, P<0.01, \mathrm{n}=12$ ). There was no significant difference in core temperature recorded at $22.00 \mathrm{~h}$ (arthritic rats,

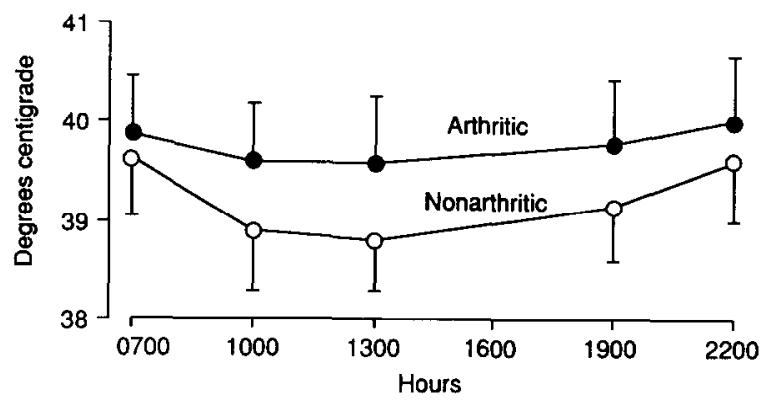

Fig. 2. Changes in core body temperature $(X \pm S . D$.) in arthritic $(\bullet, n=11)$ and non-arthritic $(O, n=12)$ rats. $40.0^{\circ} \mathrm{C} \pm 0.664$ and non-arthritic rats, $39.6^{\circ} \mathrm{C} \pm$ $0.61, P>0.05$, Fig. 2).

\section{Discussion}

Experimentally induced arthritis in the rat produces a fragmented pattern of sleep and wakefulness. During both the time of maximal sleep $(08.00-11.00 \mathrm{~h})$ and maximal wakefulness (20.00-23.00 h), arthritic rats show large increases in the number of sleep bouts and episodes of wakefulness. The increase in episodes of wakefulness observed in arthritic rats is consistent with prior reports of an increase in the number of awakenings in patients with rheumatoid arthritis $[27,30]$. A fragmented pattern of sleep is further manifested in arthritic rats by a considerable reduction in the duration of time spent asleep (sleep bouts) and in the duration of episodes of high-amplitude NREM sleep 2 (slow-wave sleep) during the time of maximal sleep in control rats.

These findings from EEG recordings of sleep patterns in arthritic rats support previous behavioral observations reporting that arthritic rats engage in more prodromal sleep behaviors [17]. Thus, arthritic rats are repeatedly aroused during the hours of maximal sleep and they are more sleepy - as indicated by significantly more sleep bouts and episodes of high-amplitude NREM sleep 1 - 
during the hours of maximal wakefulness. Fragmented patterns of sleep represent a loss in the continuity of sleep and are less restorative than non-fragmented sleep [3-5].

Moldofsky et al. [30] hypothesized that pain is the cause of disrupted sleep patterns in patients with rheumatoid arthritis, and that this non-restorative sleep, in turn, is the cause of the increase in pain and tenderness reported by patients in the morning. The possibility that pain is the cause of disrupted sleep in arthritic rats is suggested not only by the fact that arthritic rats demonstrate behavioral signs of chronic pain, but also by the fact that the elimination of pain in these rats reduces the signs of morbidity [14].

Considerable evidence exists that adjuvant arthritis in the rat is associated with chronic pain [7,8]. Arthritic rats lose weight $[7,11,12,14,17]$, hyperventilate $[11,12]$, reduce activity $[7,14,17]$, and become irritable and hyperreactive when handled [33]. In these rats there is a reduction in vocalization and withdrawal threshold to mechanical paw pressure [7,23] and to slight flexion and extension of inflamed joints [7,26]. The elimination of most of the pain in arthritic rats by surgical lesions of the 'pain transmission' pathways of the spinal cord, which elevates vocalization thresholds, attenuates the weight loss and decreased activity [14]. Arthritic rats also select and develop a preference for solutions containing non-steroidal antiinflammatory or low doses of opiate analgesics $[9,10]$. The intake of the opiate solution correlates with the time course of pain responses as measured by a change in vocalization threshold [10]. Thus, pain is a critical factor producing morbidity in arthritic rats and also may be a cause of fragmentation in sleep patterns.

Two concomitant aspects of arthritis, immobility [39] and elevated temperature [20,21], also may explain, in part, the fragmentation in sleep patterns observed in arthritic rats. Healthy humans placed at bed rest develop more fragmented sleep with frequent awakenings and shorter sleep stage episodes. These findings, however, are not consistent in all studies [see 39 for review]. In fact, the results from 2 studies of young healthy adults confined to bedrest under a normal day-night cycle showed just the opposite, immobility decreased episodes of wakefulness and increased the number and duration of episodes of NREM and REM sleep [31,36].

Following the administration of pyrogens, both increases and decreases in slow-wave sleep and REM sleep have been reported $[19,20,22,24,25,28$, $37,38]$. The decreases in these stages of sleep and an increase in the number of awakenings were associated with the peak of the febrile response $[20,22,38]$. When the elevation of body temperature was less than $1^{\circ} \mathrm{C}$, there were, in fact, no significant alterations in sleep patterns $[20,22,25$, 28 . These data suggest that the small elevation of core body temperature observed in arthritic rats in this study would have minimal effects upon sleep.

In summary, experimentally induced arthritis in the rat, a model of chronic pain, causes a marked fragmentation of sleep and a loss of the diurnal variations of sleep and wakefulness. Interventions that reduce pain associated with arthritis may reduce the fragmentation in the sleep patterns of arthritic rats. We are currently evaluating the effects of several analgesics on sleep patterns in the arthritic rat.

\section{Acknowledgements}

This work was supported by NIH Grant AM32634 to Dr. Levine, and by an ANF grant, 'Bristol-Myers Fund Scholar,' to Ms. Landis. We are grateful to Dr. Clyde Helms for reading the radiographs and to Drs. Allan Basbaum and Terrence Coderre for their helpful suggestions in the preparation of the manuscript.

\section{References}

1 Ackerman, N.R., Rooks, II, W.H., Shott, L., Genant, H, Maloney, P. and West, E., Effects of naproxen in connective tissue changes in the adjuvant arthritic rat, Arthr. Rheum., 22 (1979) 1365-1374.

2 Bergmann, B.M., Winter, J.B., Rosenberg, R.S. and Rechtschaffen, A., NREM sleep with low-voltage EEG in the rat, Sleep, 10 (1987) 1-11.

3 Bonnet, M.H., Effect of sleep disruption on sleep, perfor mance and mood, Sleep, 8 (1985) 11-19.

4 Bonnet, M.H. Performance and sleepiness as a function of 
frequency and placement of sleep disruption, Psychophysiology, 23 (1986) 263-271.

5 Bonnet, M.H., Sleep restoration as a function of periodic awakening, movement, or electroencephalographic change, Sleep, 10 (1987) 364-373.

6 Borbély, A.A. and Neuhaus, H.U., Sleep-deprivation effects on sleep and EEG in the rat, J. Comp. Physiol., 133 (1979) 71-87.

7 Calvino, B., Crepon-Bernard, M. and Le Bars, D., Parallel clinical and behavioral studies of adjuvant arthritis in the rat: possible relationship with 'chronic pain,' Behav. Brain Res., 24 (1987) 11-29.

8 Colpaert, F.C., Evidence that adjuvant arthritis in the rat is associated with chronic pain, Pain, 28 (1987) 201-222.

9 Colpaert, F.C., De Witte, Ph., Mondi, A.N., Awouters, F., Niemegeers, C.J.E. and Janssen, P.A.J., Self-administration of the analgesic suprofen in arthritic rats: evidence of Mycobacterium butyricum-induced arthritis as an experimental model of chronic pain, Life Sci., 27 (1980) 921-928.

10 Colpaert, F.C., Meert, Th., De Witte, Ph. and Schmitt, P., Further evidence validating adjuvant arthritis as an experimental model of chronic pain in the rat, Life Sci., 31 (1982) 67-75.

11 Colpaert, F.C. and Van den Hoogen, R.H.W.M., Ventilatory response to adjuvant arthritis in the rat, Life Sci., 32 (1983) 957-963.

12 Colpaert, F.C. and Van den Hoogen, R.H.W.M., Time course of the ventilatory response to adjuvant arthritis in the rat, Life Sci., 33 (1983) 1065-1073.

13 Committee for Research and Ethical Issues of the I.A.S.P., Ethical standards for investigations of experimental pain in animals, Pain, 9 (1980) 141-143.

14 Dardick, S.J., Basbaum, A.I. and Levine, J.D., The contribution of pain to disability in experimentally induced arthritis, Arthr. Rheum., 29 (1986) 1017-1922.

15 De Castro, J.M., Diurnal rhythms of behavioral effects on core temperature, Physiol. Behav., 21 (1978) 883-886.

16 De Castro, J.M. and Brower, E., Brief communication: simple, reliable and inexpensive telemetry system for continuous monitoring of small animal core temperature, Physiol. Behav., 19 (1977) 331-333.

17 De Castro Costa, M., De Sutter, P., Gybels, J. and Van Hees, J., Adjuvant-induced arthritis in rats: a possible model of chronic pain, Pain, 10 (1981) 173-185.

18 Hirose, $K$. and Jyoyama, $H$., Measurement of arthritic pain and effects of analgesics in the adjuvant-treated rats, Jap. J. Pharmacol., 21 (1971) 717-720.

19 Inoue, S., Honda, K., Komoda, K., Uchizono, K., Ueno, R. and Hayaishi, O., Differential sleep-promoting effects of five sleep substances nocturnally infused in unrestrained rats, Proc. Natl. Acad. Sci. (USA), 81 (1984) 6240-6244.

20 Kadlecova, O., Masek, K., Raskova, H. and Rotta, J., Fever and sleep cycle impairment after streptococcal mucopeptide administration, Toxicon, 10 (1972) 473-477.

21 Kales, A. and Kales J., Evaluation and Treatment of Insomnia, Oxford University Press, New York, 1984.

22 Karacan, I., Wolff, S.M., Williams, R.L., Hursch, C.J. and Webb, W.B., The effects of fever on sleep and dream patterns, Psychosomatics, 9 (1968) 331-339.
23 Kayser, V. and Guilbaud, G., The analgesics effects of morphine, but not those of the enkephalinase inhibitor thiorphan, are enhanced in arthritic rats, Brain Res., 267 (1983) 131-138.

24 Krueger, J.M., Davenne, D. and Sholam, S., Enhancement of slow wave sleep by monophosphoryl lipid A, Sleep Res., 15 (1986) 22.

25 Krueger, J.M., Karaszewski, J.W., Davenne, D. and Sholam, S., Somnogenic muramyl peptides, Fed. Proc., 45 (1986) 2552-2555.

26 Kuzuna, S. and Kawai, K., Evaluation of analgesic agents in rats with adjuvant arthritis, Chem. Pharmacol. Bull. (Tokyo), 23 (1975) 1184-1191.

27 Mahowald, M.W., Mahowald, M.L., Bundlie, S.R. and Ytterberg, S., Sleep fragmentation and daytime sleepiness in rheumatoid arthritis, Sleep Res., 16 (1987) 487.

28 Masek, K., Immunopharmacology of muramyl peptides, Fed. Proc., 45 (1986) 2549-2551.

29 Mistlberger, R.E., Bergmann, B., Waldenar, W. and Rechtschaffen, A., Recovery sleep following sleep deprivation in intact and suprachiasmatic nuclei-lesioned rats, Sleep, 6 (1983) 217-233.

30 Moldofsky, H., Lue, F.A. and Smythe, H.A., Alpha EEG sleep and morning symptoms in rheumatoid arthritis, J. Rheumatol., 10 (1983) 373-379.

31 Nakagawa, Y., Continuous observation of EEG patterns at night and in daytime of normal subjects under restrained conditions. I. Quiescent state when lying down. Electroenceph. Clin. Neurophysiol., 49 (1980) 524-537.

32 Pearson, C.M. and Wood, F.D., Studies of polyarthritis and other lesions induced in rats by injection of mycobacterial adjuvant. I. General clinical and pathology characteristics and some modifying factors, Arthr. Rheum., 2 (1959) 440-459.

33 Pircio, A.W., Fedele, C.T. and Bierwagen, M.E., A new method for the evaluation of analgesic activity using adjuvant-induced arthritis in the rat, Eur. J. Pharmacol., 31 (1975) 207-215.

34 Prinz, P.N. and Raskind, M., Aging and sleep disorders. In: R.L. Williams and I. Karacan (Eds.), Sleep Disorders, Diagnosis and Treatment, Wiley, New York, 1978, pp. 303-322.

35 Rosenberg, R.S., Bergmann, B.M. and Rechtschaffen, A., Variations in slow wave activity during sleep in the rat, Physiol. Behav., 17 (1976) 931-938.

36 Ryback, R.S. and Lewis, O.F., Effects of prolonged bedrest on EEG sleep patterns in young, healthy volunteers, Electroenceph. Clin. Neurophysiol, 31 (1971) 395-399.

37 Tobler, I., Borbély, A., Schwyzer, M. and Fontana, A., Interleukin-I derived from astrocytes enhances slow wave activity in sleep EEG of the rat, Eur. J. Pharmacol., 104 (1984) 191-192.

38 Ueno, R., Matsumura, H., Hayaishi, O., Fujita, I., Nishino, H. and Oomura, Y., Regulation of sleep by prostaglandins in conscious rhesus monkeys, Sleep Res., 16 (1987) 36.

39 Winget, C.M. and Deroshia, C.W., Psychosocial and chronophysiological effects of inactivity and immobilization. In: H. Sandler and J. Vernikos (Eds.), Inactivity: Physiological Effects, Academic Press, New York, 1986, pp. 123-147. 